\title{
Whitening of Sidelobe Powers by Pattern Switching in Radar Array
}

\section{Antenna}

\author{
M. SACHIDANANDA, RICHARD J. DOVIAK, SENIOR MEMBER, IEEE, AND DUSAN S. ZRNIĆ, SENIOR MEMBER, IEEE
}

\begin{abstract}
Presented here is a concept for nearly eliminating bias errors in reflectivity and velocity estimates due to power received through antenna sidelobes of pulse Doppler radars. The antenna pattern is switched from pulse to pulse among specially designed patterns, with near identical main lobes, but with sidelobes having randomly distributed phases and amplitudes. The sidelobe signal then becomes incoherent without affecting the coherency of the main lobe signal. The whitened sidelobe signal does not bias the mean velocity estimate computed by Doppler processing, and an unbiased estimate of reflectivity can be computed with the knowledge of the mean whitened power level. Pattern design criteria and a method for the design of optimum patterns are developed for a linear array. The extent of sidelobe reduction by way of whitening has been studied in detail for special case of only two patterns switched randomly using a pseudonoise sequence. Pattern switching realizes an effectively low sidelobe pattern without sacrifice of main lobe resolution. A possible extension to twodimensional arrays is suggested.
\end{abstract}

\section{INTRODUCTION}

$\mathbf{P}$ ULSED DOPPLER radar used for weather observation can estimate the three important spectral moments which provide information about the reflectivity, velocity and turbulence within its resolution volume. Unlike aircraft or missile detection, the measurement capability required of a weather radar imposes a more severe constraint on the antenna sidelobe level, mainly because of the distributed nature of the scatterers and their large dynamic range $(80 \mathrm{~dB})$. Furthermore, the extent of solid angle covered by the scatterers can be several orders of magnitude larger than the solid angle encompassed by the antenna main lobe. Therefore, because the echo power is proportional to the integral of the reflectivity field weighted by the two-way radar antenna pattern, the power received through the sidelobes can be comparable to that in the main lobe for weather targets of large extent, especially when the resolution volume is in a weak reflectivity region. If the spatial distribution of hydrometeor velocities and reflectivities are such that targets from sidelobes produce white spectra, the reflectivity, mean velocity, and Doppler spectrum width can be estimated provided the integrated power through sidelobes is comparable or less than the power received through the main lobe. But too often antenna sidelobes couple powerful coherent signals into the Doppler receiver and these cannot be easily discriminated from the main lobe signals. These then bias the spectral moment estimates and may cause erroneous interpretation of storm structure. Fig. 1 shows an example of a Doppler spectrum heavily contaminated by echoes received through sidelobes; the beam is in a low reflectivity region [1]. Because we have accurate velocity estimates in regions of high reflectivity in the neighborhood of the resolution volume which produced the spectrum on Fig. 1, we were able to use the continuity of velocity fields to deduce that only the peak velocity $30 \mathrm{~m} \mathrm{~s}^{-1}$ is contributed via the main

Manuscript received July 10, 1984; revised January 22, 1985.

The authors are with the National Severe Storms Laboratory, 1313 Hallev Circle, Norman, OK 73069.

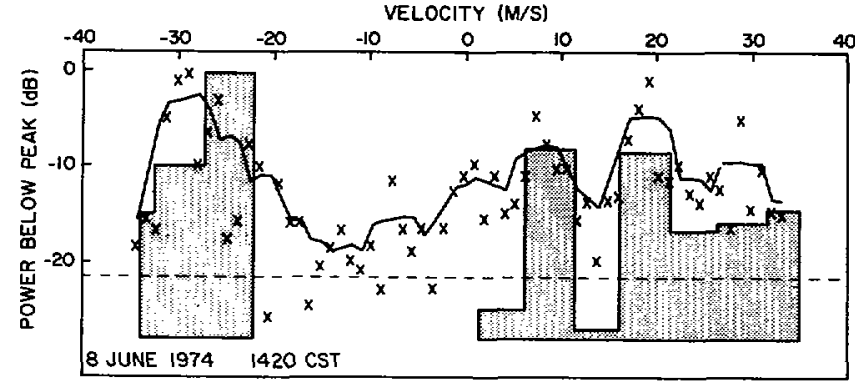

Fig. 1. Power spectrum obtained with a pulsed Doppler radar viewing a weak $(<25 \mathrm{dBZ})$ reflectivity region in a storm. The antenna main lobe has a $3 \mathrm{~dB}$ one-way beamwidth $\theta_{1}=0.8^{\circ}$, the range depth of the resolution volume (6 $\mathrm{dB}$ width) is $180 \mathrm{~m}$. The resolution volume is located at $5.1^{\circ}$ elevation, $24^{\circ}$ azimuth and $52 \mathrm{~km}$ slant range. Spectral powers are marked with $x$ and a five-point running average is drawn for visual clarity. Shaded region represents calculated sidelobe power spectra.

lobe. Through the use of radial velocity and reflectivity distributions in azimuth and elevation (at a constant range) in high reflectivity regions and the antenna pattern we estimated in the sidelobe Doppler spectrum shown by the shaded areas in Fig. 1. Although our calculation is rather crude, it can reproduce most of the observed spectral peaks. Note that because in this example three spectral peaks have magnitudes within $5 \mathrm{~dB}$ of the genuine peak (at $30 \mathrm{~m} \mathrm{~s}^{-1}$ ) it is extremely difficult to eliminate their contribution to the spectral moments automatically.

Gust fronts, downbursts, etc., can be in the regions of weak reflectivity and if there the average radial velocity is different from those illuminated by the sidelobes intersecting high reflectivity regions, the mean velocity estimates will be badly biased. To reduce the bias errors in the estimation of reflectivity and mean Doppler velocity, the usual procedure is to design the antenna with as low sidelobes as possible.

In practice, antenna design is a trade-off between the two conflicting requirements of narrow beamwidth and low sidelobe level. The reduction of bias error in the velocity estimate by reducing antenna sidelohe would necessarily result in a loss of angular resolution due to increased beamwidth. This paper presents a technique to reduce the velocity bias error without recourse to low sidelobe design so that the fine resolution is retained.

In order to estimate the Doppler spectral moments, the Doppler radar uses a coherent detection process to recover the amplitude and phase of the received signal. If the phases of the received signal are random as in a noncoherent radar, Doppler processing would result in a white noise spectrum. The technique used here is to make the signals received through the sidelobes incoherent while retaining the main lobe coherency. The antenna pattern is switched from pulse to pulse among a set of specially designed patterns which have nearly identical main lobes but sidelobes differing in phases. If the two-way radar antenna 
pattern phases are distributed uniformly over the entire span 0 , $2 \pi$ at every angle in the sidelobe region, a random switching of patterns would effectively whiten the power received through the sidelobes without affecting the main lobe power.

Whereas the whitened sidelobes would not bias spectral moments they would increase the standard error of these estimates. But because velocity fields are generally continuous some averaging in range and azimuth would reduce random errors. Furthermore, the technique developed in this paper allows considerable filtering of sidelobe power, details of which will be explored in the near future.

A method for the design of antenna patterns for a switched pattern pulsed Doppler radar has been developed. The method is applied to a linear array to demonstrate the technique. A special case of only two patterns switched randomly has also been investigated, in an effort to simplify the realization of the scheme.

\section{DOPPLER RADAR SIGNAL}

Meteorological targets consist of a large number of hydrometeor scatterers spread over a large area. The $m$ th sample voltage $V\left(\tau_{s}, m\right)$ measured by the Doppler radar, expressed as a summation of the contributions from all the scatterers [2] , is

$$
\begin{aligned}
V\left(\tau_{s}, m\right)= & \sum_{i}\left|A_{i}\right| \lg \left(\theta_{i}, \phi_{i}\right) \mid \exp \left[j \frac{4 \pi r_{i}}{\lambda}\right. \\
& \left.+\frac{4 \pi m T v_{i}}{\lambda}+\psi_{i}+2 \xi\left(\theta_{i}, \phi_{i}\right)\right]
\end{aligned}
$$

where $\tau_{s}$ is the range time, $m T$ is the sample time with $T$ as the pulse repetition period and $\left(r_{i}, \theta_{i}, \phi_{i}\right)$ is the location of the $i$ th scatterer of radar cross section $\sigma_{i}$ moving with a radial velocity $v_{i}$.

$$
A_{i}=\left[\frac{\lambda^{2} l^{2} \sigma_{i} W\left(r_{i}\right)}{(4 \pi)^{3} r_{i}^{4}}\right]^{1 / 2} .
$$

The wavelength is $\lambda, l$ is the path attenuation, and $W\left(r_{i}\right)$ is the range weighting function which depends on the pulse width $\tau$ and receiver bandwidth. $g(\theta, \phi)=P_{t}^{1 / 2} G f^{2}(\theta, \phi)$ is the antenna pattern function where $P_{t}$ is the radiated power, $G$ the power gain, and $f(\theta, \phi)$ is the complex normalized far-field pattern. $\xi(\theta, \phi)$ is the phase of $f(\theta, \phi)$. The phase $\psi_{i}$ contains the phase of the range weighting function and the phase shift at the target.

Doppler radar recovers the phases and amplitudes of these voltage samples to calculate the mean Doppler velocity and reflectivity of the resolution volume at range $r_{0}$ corresponding to the range time $\tau_{s}$. While sampling of the signals in range is done by the range gate positioned at delay time $\tau_{s}$, sampling in angular direction $(\theta, \phi)$ is done by directing the antenna beam. In order that the voltage sample $V\left(\tau_{s}, m\right)$ represents truly the signal return from the resolution volume, the antenna pattern has to be ideally a sector beam pattern with a beamwidth equal to the one way $3 \mathrm{~dB}$ beamwidth $\theta_{1}$. But in practice the antenna pattern has sidelobes over a large solid angle and the power received through the sidelobes biases the reflectivity and velocity estimates.

\section{CONCEPT OF SWITCHED PATTERN DOPPLER RADAR}

We consider here mean velocity estimation based on discrete Fourier transform (DFT) of time samples. Mean Doppler fre- quency is estimated using the power spectrum $\hat{P}\left(\tau_{s}, k\right)$ estimates computed by DFT processing $M$ samples. Thus,

$$
\hat{P}\left(\tau_{s}, k\right)=\left|\frac{1}{M} \sum_{m=0}^{M-1} V\left(\tau_{s}, m\right) e^{-j 2 \pi m k / M}\right|^{2} .
$$

Substituting (1) in (2) and rearranging the terms, the power spectrum for an unswitched pattern is

$$
\begin{aligned}
\hat{P}_{u}\left(\tau_{s}, k\right)= & \left|\sum_{i}\right| A_{i} \mid \exp \left[j \frac{4 \pi r_{i}}{\lambda}+\psi_{i}\right] g\left(\theta_{i}, \phi_{i}\right) \frac{1}{M} \\
& \left.\cdot \sum_{m=0}^{M-1} \exp \left[j \frac{4 \pi m T v_{i}}{\lambda}-2 \pi m k / M\right]\right|^{2} .
\end{aligned}
$$

The scatterer velocity $v_{i}$ is assumed to be constant over the time interval $(M-1) T$.

Consider a scheme where the antenna pattern is switched from pulse to pulse, making $g(\theta, \phi)$ a function of sample time $m T$. Denoting the patterns by $g_{m}(\theta, \phi)=P_{t m}^{1 / 2} G_{m} f_{m}^{2}(\theta, \phi)$, the power spectrum for the switched pattern is

$$
\begin{aligned}
\dot{P}_{s}\left(\tau_{s}, k\right)= & \left|\sum_{i}\right| A_{i} \mid \exp j\left[\frac{4 \pi r_{i}}{\lambda}+\psi_{i}\right] \frac{1}{M} \\
& \left.\cdot \sum_{m=0}^{M-1} g_{m}\left(\theta_{i}, \phi_{i}\right) \exp \left[j \frac{4 \pi m T v_{i}}{\lambda}-2 \pi m k / M\right]\right|^{2} .
\end{aligned}
$$

Because the antenna pattern is under the control of the designer, direction sensitive changes could be introduced into the received signal such that the resulting power spectrum $\hat{P}_{s}\left(\tau_{s}, k\right)$ gives an unbiased estimate of Doppler velocity of targets in the main lobe.

In order to characterize the effect that pattern switching will have on the Doppler spectrum, assume that all the targets in an elemental volume along the $(\theta, \phi)$ direction and within a spherical shell $c r / 2$ thick at $r=c \tau_{s} / 2$ have the same radial velocity, $v_{0}=$ $\lambda k_{0} / 2 T M$, where $k_{0}$ is an integer. Although the assumption of perfectly coherent element of sidelobe power is idealized, it will serve our purpose to characterize the effect of pattern switching. If the pattern is not switched all the elemental sidelobe power will be contained in a single spectral line $\hat{P}_{u}\left(\tau_{s}, k_{0}\right)$; the spectral power at the other lines being zero. Now, if the patterns are switched the elemental sidelobe power will spread to other spectral lines $k \neq k_{0}$ while reducing the power at $k=$ $k_{0}$. The distribution of power is now $\hat{P}_{s}\left(\tau_{s}, k\right)$. The manner in which the spectrum of the echo power received from a given direction $(\theta, \phi)$ is redistributed over the entire Nyquist interval $\lambda / 2 T$ is determined by the spectrum of the samples $g_{m}(\theta, \phi)$.

The design criteria for the antenna patterns and the switching sequence are derived based on the fact that the velocity estimate from a white noise signal is random. If we design the antenna patterns $f_{m}(\theta, \phi)$ and the switching sequence such that the spectrum of the samples $g_{m}(\theta, \phi)$ is white for all $(\theta, \phi)$ in the sidelobe region; the redistributed sidelobe power will not bias the mean velocity estimate but only increase the estimate variance. In order to retain the coherency of the main lobe signal, the main lobes of all the patterns have to be the same. We can express 
these criteria in terms of the spectrum of the samples $g_{m}(\theta, \phi)$ as

$$
\left.\begin{array}{rlrl}
S(\theta, \phi, l) & =C_{1}, & & l=0 \\
=0, & & l \neq 0
\end{array}\right\} \begin{gathered}
(\theta, \phi) \text { in the main lobe } \\
\text { region }
\end{gathered}
$$

and

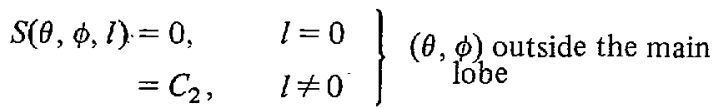

where

$$
S(\theta, \phi, l)=\left|\frac{1}{M} \sum_{m=0}^{M-1} g_{m}(\theta, \phi) e^{-j 2 \pi l m / M}\right|^{2}
$$

and $C_{1}$ and $C_{2}$ are constants. The criterion (5a) is satisfied only when the main lobes of all the patterns are identical. Since the main lobe gain and pattern shape are coupled, any change in $f(\theta, \phi)$ changes this gain. To offset this change we need to change the transmitted power $P_{t}$, hence the inclusion of $P_{t m}^{1 / 2}$ in the expression for $g_{m}(\theta, \phi)$.

The criterion (5b) implies that $S(\theta, \phi, l)$ is nearly white because $C_{2}$ is independent of $l$ except at $l=0$. The criterion that $S(\theta, \phi, 0)$ is zero sets the coherent component of the elemental sidelobe power to zero. One way to satisfy this criterion is to choose all $\left|g_{m}(\theta, \phi)\right|$ equal and the phases $2 \xi_{m}(\theta, \phi)$ uniformly spread over $(0,2 \pi)$. These patterns are switched in a random sequence to whiten the sidelobe spectrum. However, in practice it is not possible to satisfy the criterion exactly. Depending on the accuracy to which $(5 \mathrm{~b})$ is satisfied for $l=0$, some amount of sidelobe power still remains at the index $k=k_{0}$ and is given by $\hat{P}_{s}\left(\tau_{s}, k_{0}\right)$.

Putting $k=k_{0}$ in (4) we have the coherent component of sidelobe power retained at the $k_{0}$ th spectral index ar

$$
\begin{aligned}
\hat{P}_{s}\left(\tau_{s}, k_{0}\right)= & \mid \sum_{i}\left(A_{i}\right) \exp \left(j \frac{4 \pi r_{i}}{\lambda}+\psi_{i}\right) \\
& \left.\cdot \frac{1}{M} \sum_{m=0}^{M-1} g_{m}(\theta, \phi)\right|^{2} .
\end{aligned}
$$

Comparing this expression to one corresponding to the unswitched pattern, we can define an effective pattern for power at $k_{0}$ as

$$
g_{\text {eff }}(\theta, \phi)=\frac{1}{M} \sum_{m=0}^{M-1} g_{m}(\theta, \phi) .
$$

Thus, the gain patterns $g_{m}(\theta, \phi)$ of the switched array give the same coherent power at the $k_{0}$ th index as an unswitched array with pattern $g_{\text {eff }}(\theta, \phi)$. Because the unswitched array does not alter the coherency of the echoes we shall refer to the residual power at the $k_{0}$ th index as the coherent component and thus its magnitude gauges the effectiveness of the switched array to spread power into other spectral locations.

Although Doppler radar echoes are usually correlated from pulse to pulse, switching causes those received through sidelobes to be uncorrelated. The estimate of mean echo power (proportional to gain squared) from the sidelobe region can be computed using an equivalent root mean square (rms) pattern defined as

$$
g_{\mathrm{rms}}(\theta, \phi)=\left[\frac{1}{M} \sum_{m=0}^{M-1}\left|g_{m}(\theta, \phi)\right|^{2}\right]^{1 / 2} .
$$

This is an equivalent unswitched pattern which will be used to determine how well we have whitened the sidelobe power by pattern switching.

If the criteria (5) are satisfied all the sidelobe power appears as white noise while the main lobe spectrum remains unaffected. Because it is possible to estimate the level of this white noise [3] it can be subtracted from each of the computed spectral elements to obtain only the main lobe spectrum. Then both the reflectivity and mean velocity estimates, computed using the emended spectrum, will be completely unbiased.

While this suggests that we can use patterns with higher sidelobes to take advantage of their narrower main lobe and higher gain, too high sidelobes will affect the accuracy of reflectivity and mean velocity estimates. If we consider sidelobe power as noise, the signal to noise ratio of the switched pattern Doppler signal is approximately equal to main lobe to sidelobe signal power ratio (assuming the receiver noise power is small compared to the sidelobe echo power). It is necessary to keep this ratio high enough so that the accuracy of reflectivity and velocity estimates it not sacrificed in the process of removing the bias error.

Assuming a uniform reflectivity field, the total mean power $P_{r s}$ received through the sidelobes can be computed using the equivalent unswitched pattern $g_{\text {rm } s}$. Thus

$$
P_{r s}=C \int_{0}^{2 \pi} \int_{\theta_{B}}^{\pi} g_{\mathrm{rms}}^{2}(\theta, \phi) \sin \theta d \theta d \phi
$$

where $C$ is a proportionality constant and $\theta_{B}$ is the first zero crossing of the pattern (assuming a pattern symmetry in $\theta$ ).

After Doppler processing, the portion of $P_{r s}$ which remains coherent can be computed using the equivalent unswitched pattern $g_{\text {eff. }}$ The portion of the coherent power $P_{c s}$ received through the sidelobes is given by

$$
P_{C S} \doteq C \int_{0}^{2 \pi} \int_{\theta_{B}}^{\pi}\left|g_{\mathrm{ef} f}(\theta, \phi)\right|^{2} \sin \theta d \theta d \phi .
$$

A measure of the effectiveness of the technique can be represented as the average sidelobe power whitening ratio $B_{v}$.

$$
B_{v}=10 \log \left(\frac{P_{c s}}{P_{r s}}\right) \mathrm{dB}
$$

This definition, of course, assumes that the redistributed sidelobe power has white noise spectrum. If the received sidelobe power is equally distributed over the entire Nyquist interval by random switching, the power in each spectral component would be $P_{r s}$ $M-1$ for a $M$ sample Doppler processor. We can expect the velocity bias error to be nearly eliminated for a whitening ratio of $B_{v}=10 \log (1 / M) \mathrm{dB}$.

It is not necessary to have $M$ different patterns for $M$ sample pulse Doppler processing. The $M$ samples can be obtained by switching only $K$ patterns $(K<M)$ in a random fashion.

\section{DESIGN OF PATTERNS}

In practice it is not possible to satisfy the criteria (5) exactly. The design is an exercise in selecting the patterns that conform to the criteria at least approximately. To illustrate the switched pattern technique we consider an example of a linear array whose excitation can be easily controlled to generate several patterns. Array excitation is assumed to be controllable 
using programmable gain amplifiers and phase shifters in the feed line of each element.

Consider a uniformly spaced broadside array of $2 N$ elements with complex excitation coefficients $A_{n}$. The normalized field pattern of the array can be expressed as (derived from basic equations in [4]),

$$
f(u)=\frac{\sum_{n=1}^{N}\left[A_{n} e^{j \psi_{n} u}+A_{-n} e^{-j \psi_{n} u}\right]}{\left|\sum_{n=1}^{N}\left(A_{n}+A_{-n}\right)\right|}
$$

where $u=\sin \theta, \theta$ measured from main lobe axis, $\psi_{n}=(2 n-1)$ $\beta d / 2, \beta=2 \pi / \lambda$ the propagation constant and $d$ is the interelement spacing. Decomposing the set of complex coefficients $A_{n}$ into real symmetric $a_{n}$, real antisymmetric $b_{n}$, imaginary symmetric $c_{n}$ and imaginary antisymmetric $d_{n}$ components.

$$
A_{n}=\left(a_{n}+b_{n}\right)+j\left(c_{n}+d_{n}\right)
$$

where $a_{n}, b_{n}, c_{n}$, and $d_{n}$ are all real, the pattern can be expressed as

$$
f(u)=\frac{\sum_{n=1}^{N}\left[\left(a_{n}+j c_{n}\right) \cos \left(\psi_{n} u\right)-\left(d_{n}-j b_{n}\right) \sin \left(\psi_{n} u\right)\right]}{\left|\sum_{n=1}^{N}\left(a_{n}+j c_{n}\right)\right|}
$$

To make the main lobes of all the $K$ patterns nearly identical, we select the symmetric coefficients $a_{n}$ to be common to all the patterns and set all the imaginary symmetric coefficients $c_{n}$ equal to zero. The antisymmetric coefficients, which produce an antisymmetric pattern about the main lobe axis, are used to spread the phase of the pattern in the sidelobe region. The field produced by $b_{n}$ and $d_{n}$ is forced to nearly zero in the main lobe region so that the main lobe remains unaffected while changing the phase of the sidelobes. The procedure followed for the synthesis of these three sets of coefficients is as follows

The symmetric coefficients $a_{n}$, which are common to all the $K$ patterns, are synthesized first. Let $h$ be the peak sidelobe level allowable for the symmetric pattern. The synthesis criterion can be either to minimize the beamwidth or to maximize the gain with a constraint on the maximum allowable sidelobe level. For a low sidelobe design these two solutions coincide, but the synthesis methods differ. We select the latter criterion and use a numerical method based on the simplex minimization algorithm [5] for the synthesis of excitation coefficients. This algorithm's capability of minimizing a broad class of functions allows incorporation of the sidelobe constraint as inequality constraints. The method can also be used when the array spacings are nonuniform or to optimize the array performance with spacings as variables.

In this method an objective function $\Phi_{a}$ for the symmetric pattern is formulated consisting of a gain function and a sidelobe constraint function, and is minimized using the simplex algorithm. To maximize the gain, negative of the gain is used in the objective function being minimized. Sidelobe level constraint at any given angle $u(u=\sin \theta)$ is written as an inequality constraint:

$$
D(u) \leqslant h, \quad u_{B}<u<1
$$

where $D(u)=\left|f_{a}(u)\right|^{2}$ is the sidelobe level with respect to the main lobe peak, and $u_{B}$ is the first zero crossing of the normalized symmetric pattern $f_{a}(u) . L$ equally spaced locations $u_{i}, i=1$, $2, \cdots, L$, are chosen to span the entire $\left(u_{B}, 1\right)$ angular sector and the sidelobe constraint function is formulated by summing the constraints at these locations. The number of locations $L$ is chosen to be greater than

$$
\left[\frac{4 d}{\lambda}(2 N-1)\left(1-u_{B}\right)\right]
$$

so that the constraint (15) is applied at least at four locations for each sidelobe. Since we do not have a priori knowledge of $u_{B}$, an approximate location of $u_{B}$ is used. It has been found that the method is not very sensitive to errors in $u_{B}$. The objective function $\Phi_{a}$ is written as

$$
\Phi_{a}=-\frac{2\left[\sum_{n=1}^{N} a_{n}\right]^{2}}{\sum_{n=1}^{N} a_{n}^{2}}+p \sum_{i=1}^{L} \max \left[0,\left(D\left(u_{i}\right)-h\right)\right] .
$$

The first term is the negative of the gain and the second, the sidelobe constraint function. The function $\max (x, y)$ choses the maximum of the two arguments and $p$ is a weighting constant used to adjust the relative weight given to each term in $\Phi_{a} . \Phi_{a}$ becomes independent of $p$ when the sidelobe constraint is satisfied at all locations $u_{i}$.

In the process of minimization it is important to choose the initial values of $p$ and $a_{n}$ judiciously to avoid solutions which are local minima. Generally, a few trial runs would be necessary to arrive at an optimum value for $p$. Coefficients corresponding to a uniform distribution would suffice as a reasonable starting point in most cases.

Initially, the antisymmetric coefficients $b_{n}$ and $d_{n}$ are generated using a random number routine. These coefficient sets are normalized such that the antisymmetric pattern peak is equal to the sidelobe constraint level $h$. Each set of these coefficients is readjusted, using the simplex algorithm, such that the resulting pattern has nearly zero field in the main lobe region $0<u<u_{B}$. The objective function $\Phi_{b}$ for the antisymmetric pattern is formulated as

$$
\Phi_{b}=\sum_{i=1}^{J} \sum_{n=1}^{N} b_{n} \sin \left(\psi_{n} u_{i}\right)
$$

where $u_{i}, i=1,2, \cdots, J$, are equally spaced locations in the main lobe region. $J$ is selected to be greater than $\left(4 u_{B}(2 N-1) d / \lambda\right)$. A similar objective function holds good for synthesis of $d_{n}$.

$K$ sets of $b_{n}$ and $d_{n}$ are generated using this procedure and the array excitation coefficients are put together for $K$ patterns.

$$
\left.\begin{array}{l}
A_{n}^{(i)}=a_{n}+w_{1}^{(i)} b_{n}^{(i)}+j w_{2}^{(i)} d_{n}^{(i)} \\
A_{-n}^{(i)}=a_{n}-w_{1}^{(i)} b_{n}^{(i)}-j w_{2}^{(i)} d_{n}^{(i)}
\end{array}\right\} \begin{aligned}
& i=1,2, \cdots, K \\
& n=1,2, \cdots, N
\end{aligned}
$$

where $w_{1}^{(i)}$ and $w_{2}^{(i)}$ are weighting constants with values between $(0,1)$ selected randomly using a random number routine.

These sets of coefficients produce $K$ normalized patterns $f_{i}(u)$ with matched mainlobes. In general, the gain and radiated power would have different values for each of these patterns. 
The phases and amplitudes in the negative $u$ direction are in general different from that in the positive $u$ direction. But it is expected that the random selection of coefficients $b_{n}$ and $d_{n}$ would produce $g_{\mathrm{rms}}$ and $g_{\text {eff }}$ patterns with same average characteristics in terms of $B_{\mathrm{v}}$ in both positive and negative $u$ directions.

It is hoped that this procedure would produce reasonably uniform spread of phases in the sidelobe region without unduly increasing the rms sidelobe level. It may be noted that the rms pattern (8) would have slightly higher sidelobes than the designed level $h$, due to the additional power in the antisymmetric patterns. In some directions this would at most be by a factor of two larger so that it can be accounted for in the overall design.

\section{RANDOM SWITCHED ARRAY SIMULATION}

To test the theory of a switched pattern array a 20 element $(N=10)$ uniformly spaced linear broadside array is considered. The interelement spacing is chosen to be $\lambda / 2$. Sixteen patterns are synthesized $(K=16)$ using the procedure of Section IV, with a sidelobe constraint level of $-30 \mathrm{~dB}$.

The one-way power patterns for the 16 set of coefficients (Fig. 2) shows that the main lobes are not affected by the addition of antisymmetric coefficients. The maximum amplitude spread in the main lobe $10 \mathrm{~dB}$ below the peak is only $0.01 \mathrm{~dB}$, but there is appreciable amplitude spread in the sidelobe region. The phases of the 16 patterns are computed at regular intervals and are presented as points in Fig. 3. Though there is reasonable spread in the phase, the distribution is not uniform. In general, the phase spread and amplitude spread are coupled, an increase in phase spread would produce an amplitude spread too.

To simulate the switched pattern scheme, the patterns are selected randomly among the 16 to generate 64 samples. Using these 64 samples, rms and effective patterns are computed (Fig. 4). There is an improvement in the sidelobe level of effective pattern compared to that of rms pattern but it is not enough to justify the complex switching scheme. The average sidelobe power whitening ratio $B_{v}$ is $-2.5 \mathrm{~dB}$. This lack of improvement in the sidelobe level is believed to be due to the nonuniformity in the phase spread and large amplitude spread of the sidelobes. In fact, due to the addition of the antisymmetric component there is an average increase of sidelobe level of approximately $2 \mathrm{~dB}$ from that of the symmetric pattern. So, if we take the sidelobe level of the symmetric pattern as the reference for comparison the improvement is only $0.5 \mathrm{~dB}$ on an average.

The complexity of the switched pattern scheme, with controlled amplifiers and phase shifters in each element, is too high a price to pay for the small improvement illustrated here. We do not know if substantially better performance is physically impossible or if our method of pattern design is not adequate.

\section{SPECIAL CASE-DUAL PATTERN ARRAY}

We consider a special case of a switched pattern scheme in an effort to simplify the realization of such an array and to improve the whitening ratio $B_{v}$. We select only two patterns $(K=2)$ and the switching is between these two patterns in a random fashion, using an $M$ bit shift register sequence. Further, we select the excitation coefficients to be completely real, eliminating the phase shifters in each element.

\section{A. Design Criteria}

For $K=2$ the pattern design criteria (5) can be modified to

$$
g_{1}(u)=g_{2}(u) ; \quad 0<u<u_{B}
$$

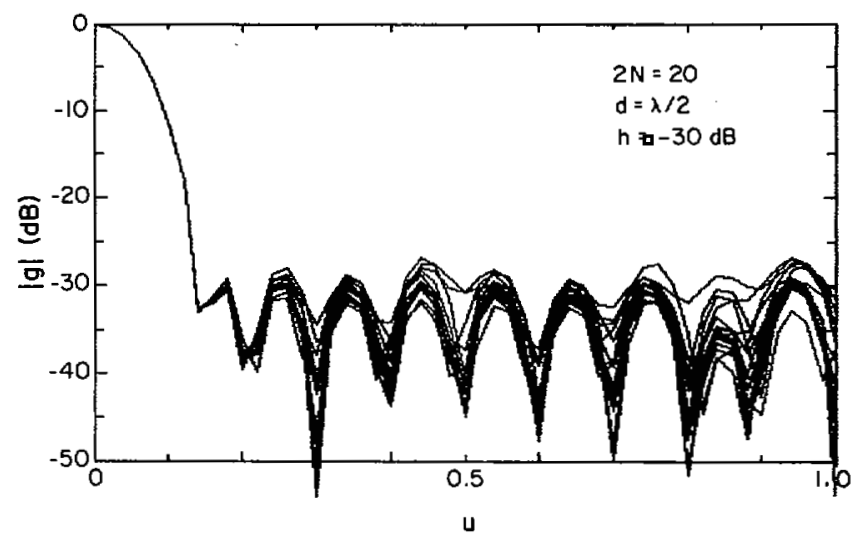

Fig. 2. Sixteen patterns for the multiple pattern switched array.

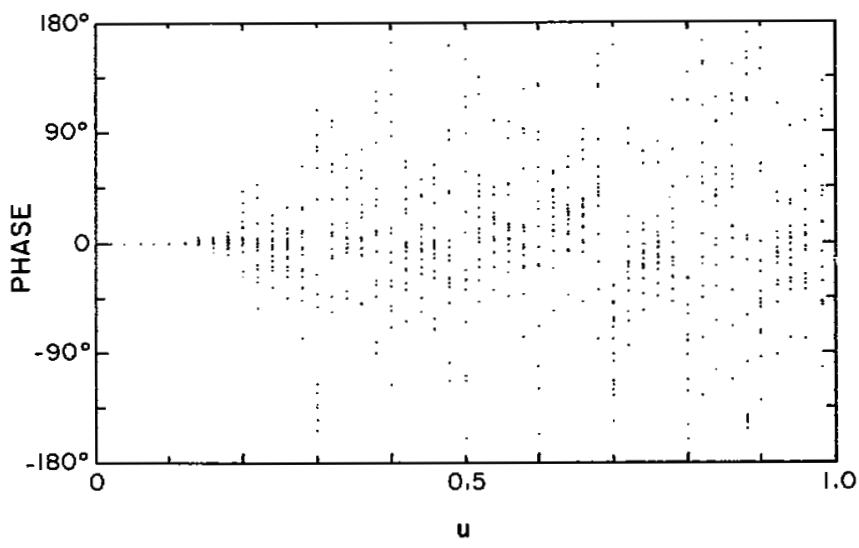

Fig. 3. Phase spread of 16 patterns.

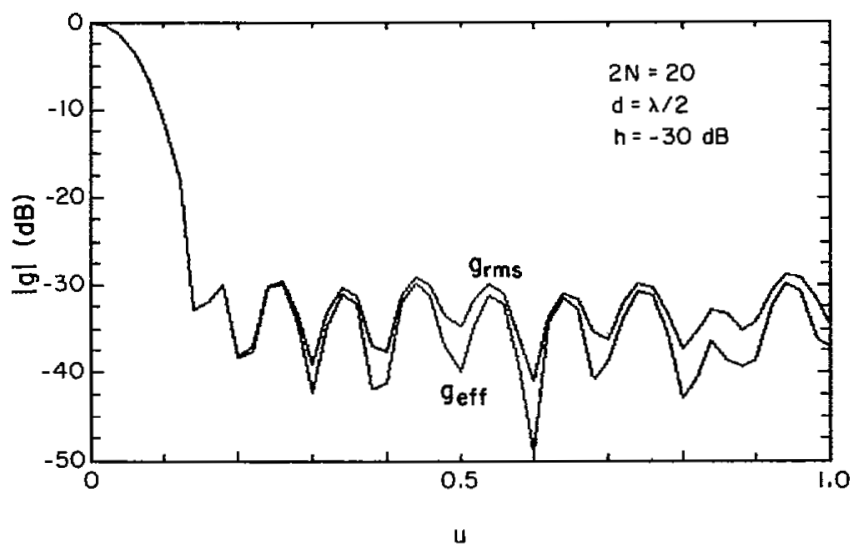

Fig. 4. RMS and effective patterns for the multiple pattern switched array.

$$
g_{1}(u)=-g_{2}(u) ; \quad u_{B}<u<1 .
$$

Since there are only two patterns to be designed a closer approximation to the design criteria (19) is possible resulting in a better whitening.

\section{B. Synthesis of Patterns}

Again, we choose the $2 \mathrm{~N}$ element uniformly spaced broadside array to illustrate the design. The real excitation coefficients $A_{n}$ are decomposed into symmetric $a_{n}$ and antisymmetric 
$b_{n}$ components

$$
\begin{aligned}
& A_{n}=a_{n}+b_{n} \\
& A_{-n}=a_{n}-b_{n}
\end{aligned}
$$

so that the complex two-way field pattern is given by

$$
\begin{aligned}
g(u) & =P_{t}^{1 / 2} G f^{2}(u) \\
& =\frac{\left[2 \sum_{n=1}^{N}\left\{a_{n} \cos \left(\psi_{n} u\right)+j b_{n} \sin \left(\psi_{n} u\right)\right\}\right]^{2}}{\left[2 \sum_{n=1}^{N}\left(a_{n}^{2}+b_{n}^{2}\right)\right]^{1 / 2}} .
\end{aligned}
$$

All the notations are the same as described earlier in Sections III and IV. If we design one pattern with coefficients $a_{n}$ and $b_{n}$, and generate the second pattern by changing the sign of antisymmetric coefficients only, the criteria (19a), (19b) can be reduced to

$$
\sum_{n=1}^{N} b_{n} \sin \left(\psi_{n} u\right)=0 ; \quad 0<u<u_{B}
$$

and

$$
\begin{aligned}
& \sum_{n=1}^{N} a_{n} \cos \left(\psi_{n} u\right)=\sum_{n=1}^{N} b_{n} \sin \left(\psi_{n} u\right) ; \\
& u_{B}<u<1 .
\end{aligned}
$$

The coefficients $a_{n}$ are synthesized as described earlier in Section III with a sidelobe constraint $h$. The procedure optimizes the gain of the symmetric pattern for a maximum allowable sidelobe level $h$.

An objective function $\Phi_{d}$ is formulated and is minimized using simplex algorithm to obtain the antisymmetric coefficients. $L$ equally spaced angular locations $u$ are chosen in the region $(0,1)$ and a reference pattern $f_{d}\left(u_{i}\right)$ is defined such that

$$
f_{d}\left(u_{i}\right)=0 ; \quad 0<u_{i}<u_{B}
$$

and

$$
f_{d}\left(u_{i}\right)=\sum_{n=1}^{N} a_{n} \cos \left(\psi_{n} u_{i}\right) ; \quad u_{B}<u_{i}<1 .
$$

The $a_{n}$ are symmetric coefficients synthesized earlier. An example of a reference pattern $f_{d}(u)$, derived from the symmetric pattern, for the synthesis of antisymmetric coefficients $b_{n}$ is shown in Fig. 5. Because the symmetric and antisymmetric patterns are in phase quadrature the effect of polarity change $\left(b_{n}=-b_{n}\right)$ is to shift the phase of $f(u)$ by $90^{\circ}$ in the sidelobe region. However, because the radar uses the same pattern in transmission and reception the change is $180^{\circ}$. Thus, the phase angle of sidelobe signal flips between $0^{\circ}$ and $180^{\circ}$.

The criteria for selecting the number $L$ is the same as the one used in Section IV. Now, the objective function $\Phi_{d}$ is expressed as

$$
\Phi_{d}=\sum_{i=1}^{L}\left|f_{d}\left(u_{i}\right)-\sum_{n=1}^{N} b_{n} \sin \left(\psi_{n} u_{i}\right)\right| \text {. }
$$

The $b_{n}$ are the variables of minimization. The minimum of this function gives a set of $b_{n}$ whose pattern is a least difference fit

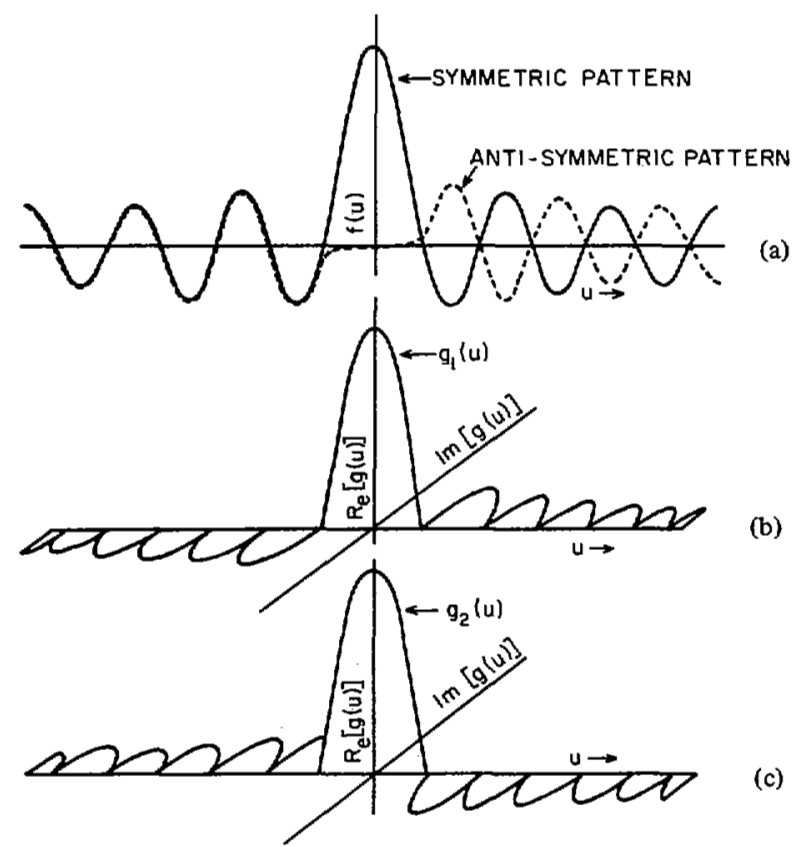

Fig. 5. Symmetric and antisymmetric design goal pattern functions and the radar two-way patterns $g_{1}(u)$ and $g_{2}(u)$ for the dual pattern switched array. The symmetric and antisymmetric patterns are in phase quadrature.

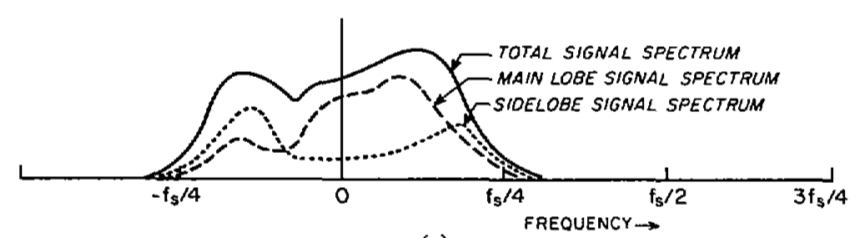

(a)

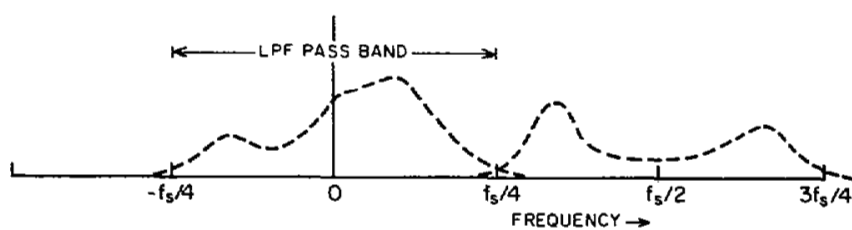

(b)

Fig. 6. Alternate pattern switching. (a) Received spectrum. (b) Spectrum after switching, for an ideal pattern design.

to the zero line in the main lobe region and to the symmetric pattern in the sidelobe region.

\section{Alternate Switching Scheme}

An interesting variant of the dual switched pattern scheme is to switch the patterns alternately, instead of using a random sequence. For an ideal pattern design satisfying the criteria (19a), (19b) the phase of the sidelobe signal only is shifted by $\pi \mathrm{rad}$ for every alternate sample, without affecting the main lobe signal. This is equivalent to shifting the sidelobe power spectrum by $f_{s} / 2$, the switching frequency. This is illustrated in Fig. 6. If the sample rate $f_{s}$ (PRF) is chosen high enough such that the entire Doppler spectrum is within $\pm f_{s} / 4$, then it is possibel to separate out the main lobe signal using a low pass filter. This can remove the bias error in the velocity estimate as well as the reflectivity estimate. Of course, the reflectivity has to be estimated from the digitized samples after filtering. A disadvantage of this scheme is its high PRF which reduces the unambiguous range. 


\section{DUAL SWITCHED PATTERN SIMULATION}

In a dual switched pattern antenna the sidelobe signal whitening is accomplished by random switching between two patterns and the effectiveness of whitening depends on how well (19b) is satisfied. The functional form in (22b) suggests that the extent of matching possible between symmetric and antisymmetric patterns in the sidelobe region would improve with larger numbers of elements in the array. Each pair of symmetrically spaced elements in the array generates its own symmetric pattern $a_{n} \cos \left(\psi_{n} u\right)$ and antisymmetric pattern $b_{n} \sin \left(\psi_{n} u\right)$ which are orthogonal to each other. We are able to match the total symmetric and antisymmetric patterns in the sidelobe tegion only because the two patterns are summations of $N$ basis functions weighted by coefficients $a_{n}$ and $b_{n}$, respectively.

Initially, a 20 element uniformly spaced broadside array with $\lambda / 2$ spacing was designed for $\mathrm{a}-30 \mathrm{~dB}$ sidelobe level. Criteria (22b) could be satisfied in most of the sidelobe region to a reasonable accuracy except in the region of first three sidelobes. The mutual orthogonality of basis functions can be disturbed by nonuniformly spacing the elements which gives us additional control in matching the symmetric and antisymmetric patterns. This is equivalent to minimizing the objective function $\phi_{d}$ with respect to $b_{n}$ as well as $\psi_{n}$ and involves formulation of a new objective function to include the practical constraint on the allowable spacings. For instance, the basic radiating elements generally have dimensions of the order $\lambda / 2$ for efficient radiation thus physically limiting the closest spacing possible. Nor is it desirable to have spacings closer than $\lambda / 2$ because of the rapidly increasing mutual coupling between the elements for spacings less than $\lambda / 2$. Further, spacings larger than $\lambda$ causes grating lobes to appear in the visible region. It may also be necessary to change the optimization strategy because of the nature of functional dependence of $\phi_{d}$ on $\psi_{n}$ and $b_{n}$. In short, further investigation is needed to evolve a satisfactory method which is effective when spacings are also treated as variables.

In the following example, we selected a set of spacings based on heuristic reasoning which gives better matching of symmetric and antisymmetric patterns in the sidelobe region at the expense of a small increase in the $-3 \mathrm{~dB}$ beamwidth. Interelement spacing is chosen to be $0.5 \lambda$ for all the elements except the end elements which are spaced $0.75 \lambda$ apart. The excitation currents are optimized with these spacings to obtain maximum possible whitening of sidelobes. The rms and effective patterns are shown in Fig. 7. A 64 bit pseudorandom sequence is used to switch the patterns in a random fashion.

Although the excitation currents are calculated with 6 digit accuracy, there is a limit to the accuracy with which element excitation can be controlled in practice. Hence, to be more realistic, the normalized excitation currents are rounded off to second decimal digit before computing the pattern in Fig. 7. The excitation coefficients are listed in Table I. It may be noted that the last two sidelobes in Fig. 7 are not whitened to the same extent as others, but these would be reduced when the array pattern is multiplied by the element pattern which generally has a null in $u=1.0$ direction.

Fig. 8 shows the sidelobe power whitening as a function of angle $u$. The superimposed rms pattern gives us an idea of the power received as a function of angle. It should be noted that all the sidelobes except the last one are whitened more than $-18 \mathrm{~dB}$, the limit beyond which a 64 sample processing would not

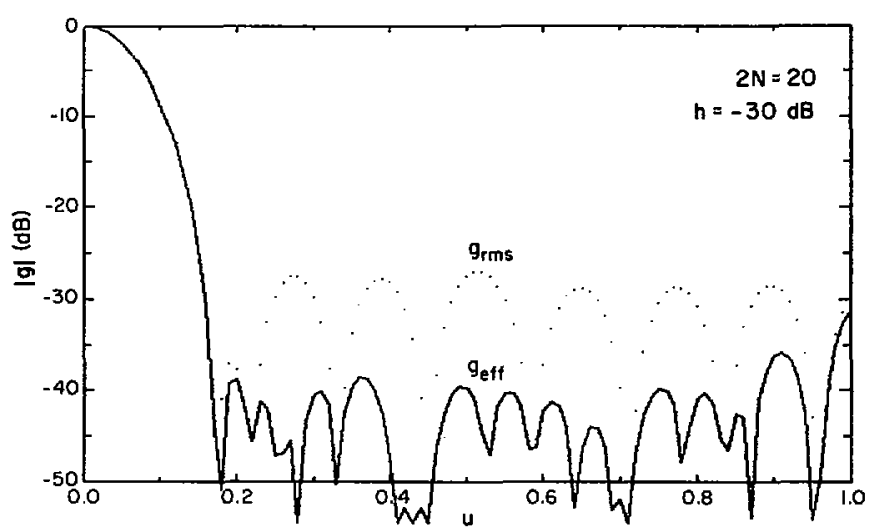

Fig. 7. $g_{\mathrm{rms}}$ and $g_{\mathrm{eff}}$ for dual pattern switched array.

TABLE I

DUAL PATTERN ARRAY COEFFICIENTS FOR A 20 ELEMENT BROADSIDE ARRAY

\begin{tabular}{lllll}
\hline \hline Spacing & $\mathrm{n}$ & $\mathrm{A}_{\mathrm{n}}$ & $\mathrm{n}$ & $\mathrm{A}_{\mathrm{n}}$ \\
\hline $0.25 \mathrm{\lambda}$ & 1 & 1.02 & -1 & 0.98 \\
0.75 & 2 & 1.00 & -2 & 0.90 \\
1.25 & 3 & 0.93 & -3 & 0.81 \\
1.75 & 4 & 0.85 & -4 & 0.71 \\
2.25 & 5 & 0.72 & -5 & 0.56 \\
2.75 & 6 & 0.59 & -6 & 0.43 \\
3.25 & 7 & 0.39 & -7 & 0.33 \\
3.75 & 8 & 0.39 & -8 & 0.37 \\
4.25 & 9 & 0.40 & -9 & 0.02 \\
5.00 & 10 & 0.12 & -10 & 0.00 \\
\hline
\end{tabular}

Spacings are from array center. $A_{n}=\boldsymbol{A}_{-n}$ for the second pattern.

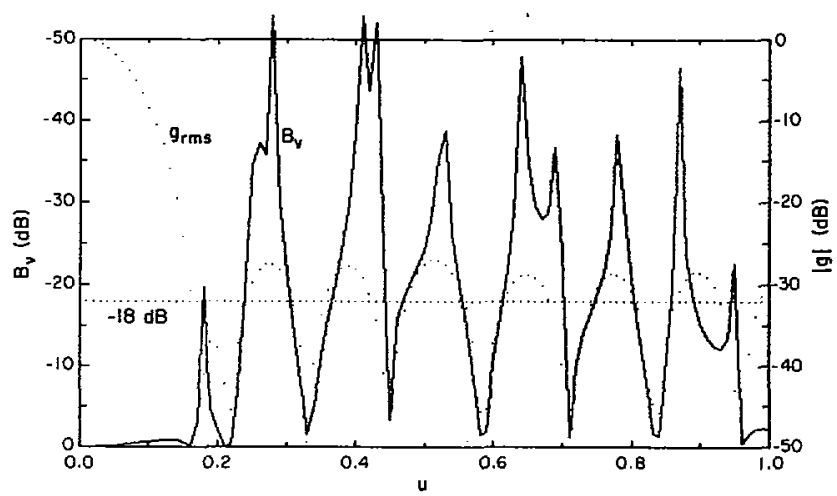

Fig. 8. Sidelobe power whitening as a function of angle.

improve the bias error reduction in velocity estimate. The minima of whitening occurs at sidelobe nulls of $g_{\mathrm{rm} \mathrm{s}}$ hence would not produce appreciable bias error. The whitening of main lobe is negligible.

The signal spectra using 64 sample DFT, presented in Fig. 9, show the redistribution of received power due to random switching. The spectra are for power received at angles $u=0.18,0.5$, and 0.92 , near where sidelobe peaks of $g_{\text {eff }}$ pattern occur. The received signal is assumed to be at zero Doppler and the spectra 


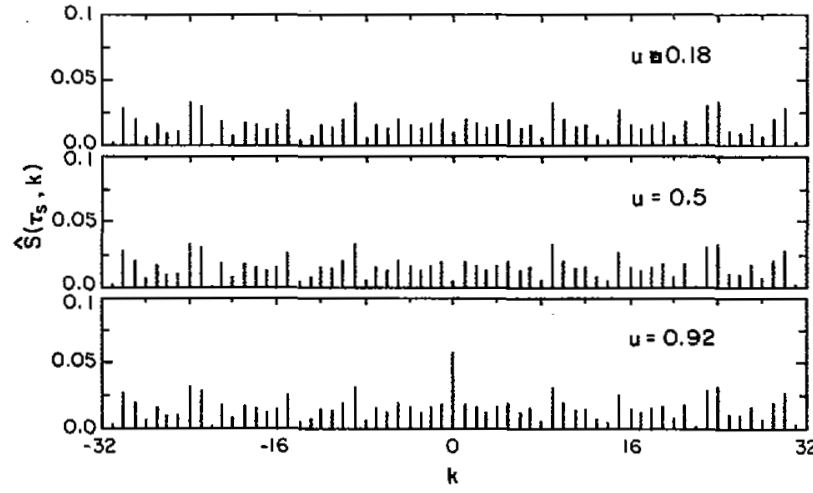

Fig. 9. Whitened spectra of the signal received from directions $u=0.18,0.5$ and 0.92 (normalized to the total power received at that angle).

are normalized with respect to the total power received in the given direction. At $u=0.92$ the whitening is approximately $-13 \mathrm{~dB}$, hence the unwhitened power in the dc component shows up above the average power level of the spectrum.

For this example the average sidelobe whitening ratio $B_{v}$ (11) is $-17.8 \mathrm{~dB}$. Fig. 7 shows that by pattern switching an effective sidelobe level of $-40 \mathrm{~dB}$ is obtained while retaining the beamwidth (BW) of a conventional $-30 \mathrm{~dB}$ sidelobe pattern. For the given spacings a conventional $-40 \mathrm{~dB}$ sidelobe pattern would give us an optimum gain of $11.65 \mathrm{~dB}$ with $3 \mathrm{~dB}$ beamwidth of $7.43^{\circ}$. A conventional $-30 \mathrm{~dB}$ sidelobe pattern design would yield an optimum gain of $12.2 \mathrm{~dB}$ with $6.51^{\circ} \mathrm{BW}$. Pattern switching yields an effective $-40 \mathrm{~dB}$ sidelobe pattern with $11.9 \mathrm{~dB}$ gain and $6.95^{\circ} \mathrm{BW}$. A little degradation in $\mathrm{BW}$ and gain from that of a conventional $-30 \mathrm{~dB}$ design' was allowed to obtain better whitening of sidelobes.

It should be noted that the above example is by no means the best possible pattern. It has been given only to demonstrate the concept of pattern switching. Patterns with better performance should be possible with nonuniform spacings.

\section{EXTENSION TO TWO-DIMENSIONAL ARRAYS}

Switched pattern principle can be easily extended to two dimensional arrays. We consider here an extension of dual pattern scheme to two-dimensional array of $4 N^{2}$ elements, and use the knowledge of excitation coefficients of a linear array of $2 \mathrm{~N}$ elements to derive the excitation coefficients of two-dimensional array with uniform spacing in $x$ and $y$ directions.

The array geometry is assumed to have a rectangular grid structure with same spacing $d_{x}$ and $d_{y}$ in $x$ and $y$ directions (Fig. 10). The excitation coefficients are represented by double subscripts indicating the location of the element with respect to the coordinate axes.

The field pattern is expressed as

$$
\begin{aligned}
& f\left(u_{x}, u_{y}\right) \\
& =\sum_{m=1}^{N} \sum_{n=1}^{N}\left[A_{m n} e^{j((2 m-1) \beta d} x^{u} x+(2 n-1) \beta d_{y} u_{y}\right) / 2
\end{aligned}
$$

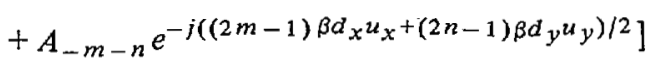

where $u_{x}=\sin \theta \cos \phi$ and $u_{y}=\sin \theta \sin \phi$, are angular pattern variables. $A_{m n}$ is a real excitation coefficient of $(m n)$ th element located at $x=(2 m-1) d_{x} / 2$ and $y=(2 n-1) d_{y} / 2$.

Let $a_{n}, b_{n}, n=1,2, \cdots, N$, be two sets of coefficients designed

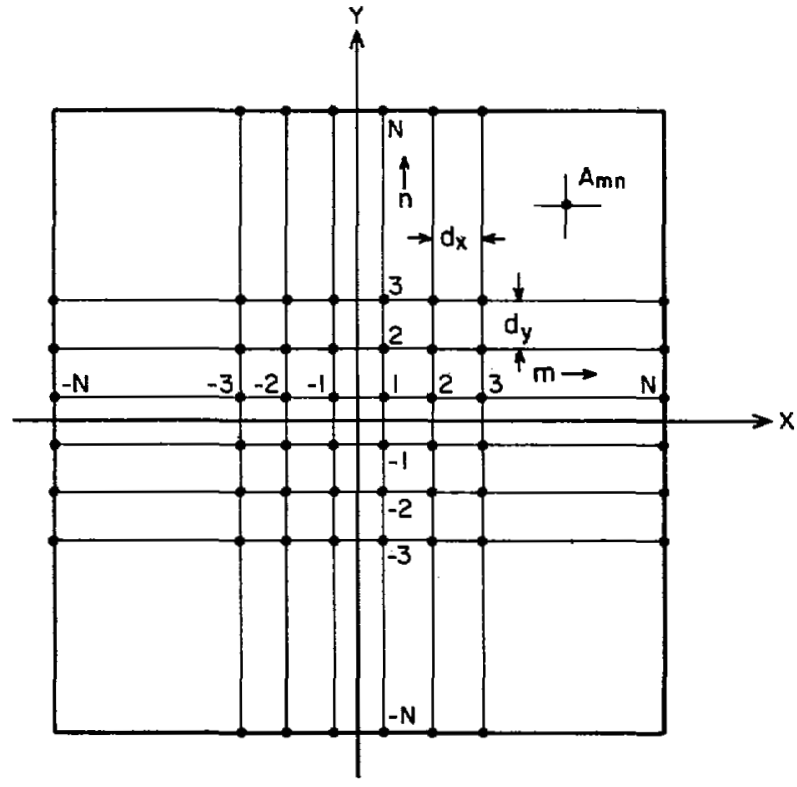

Fig. 10. Two-dimensional array geometry.

for dual pattern linear uniformly spaced array with the same spacing as the two-dimensional array, $\left(d_{x}=d_{y}\right)$. Using these coefficients the symmetric part of $A_{m n}$ can be expressed as

$$
A_{-m-n}^{s}=A_{m n}^{s}=A_{-m n}^{s}=A_{m-n}^{s}=a_{m} a_{n}
$$

Antisymmetry is introduced only in one plane. Choosing antisymmetry about $y$-axis, the antisymmetric component of $A_{m n}$ can be expressed as

$$
-A_{-m n}^{a}=A_{m n}^{a}=A_{m-n}^{a}=-A_{-m-n}^{a}=b_{m} a_{n} .
$$

For the second pattern $b_{m}$ is equal to $-b_{m}$ in (27). Hence, the two sets of coefficients for the two patterns are

$$
\begin{gathered}
A_{m n}^{(1)}=A_{m-n}^{(1)}=\left(a_{m}+b_{m}\right) a_{n} ; \\
A_{-m n}^{(1)}=A_{-m-n}^{(1)}=\left(a_{m}-b_{m}\right) a_{n} \\
A_{m n}^{(2)}=A_{m-n}^{(2)}=\left(a_{m}-b_{m}\right) a_{n} ; \\
A_{-m n}^{(2)}=A_{-m-n}^{(2)}=\left(a_{m}+b_{m}\right) a_{n} .
\end{gathered}
$$

The superscript refers to patterns 1 and 2 .

This scheme, of course, does not whiten the entire sidelobe region, but a major portion of the solid angle covered by the sidelobes is whitened, leaving a narrow region in the $u_{x}, u_{y}$ plane. The extent of sidelobe region unaffected by switching is for $u_{x}$ between $\pm u_{B}$ and $u_{y}$ between \pm 1 , where $u_{B}$ is the first zero crossing of the pattern in $u_{x}$ direction.

\section{CONCLUSION}

The concept of switched pattern pulsed Doppler radar introduced in this paper minimizes bias errors in the mean Doppler velocity estimate due to power received through the antenna sidelobes, without recourse to low sidelobe design. In this case fine resolution of the main lobe is retained. The bias error in the velocity estimate is minimized by whitening the power received through the sidelobes while maintaining coherency in the main lobe.

The technique used is to switch the antenna pattern randomly from pulse to pulse among a set of specially designed patterns. A criterion for pattern design to achieve sidelobe power whiten- 
ing without affecting the main lobe has been derived and a procedure for the design of patterns has been presented. An illustrative example of a 16 pattern switched linear array simulation showed a small improvement of $2.5 \mathrm{~dB}$ in the overall sidelobe reduction, which would not be enough to justify the complexity of the scheme. Sidelobe power whitening seems to depend heavily on the distribution of phases and amplitudes of the designed patterns in the sidelobe region. The lack of improvement can be attributed to the amplitude spread and the nonuniformity in the phase spread. We do not know whether the limitation is inherent in the pattern polynomial or in our design procedure.

A special case of only two patterns, switched randomly using a pseudorandom shift register sequence, has also been presented. Because there are only two patterns, a deterministic design rather than a random selection procedure, has been used to achieve better accuracy in satisfying the design criteria. The results of dual pattern switched array simulation are promising in terms of sidelobe whitening and its simplicity in realization. An average sidelobe whitening ratio of $-17.8 \mathrm{~dB}$ has been obtained for the example.

In our example of linear array design only excitation currents are used as variables of optimization. The spacings are selected by heuristic approach which by no means can be considered as the best. It should be possible to obtain much lower sidelobes for $g_{\text {eff }}$ by optimizing the spacings as well.

The switched pattern technique can easily be extended to twodimensional arrays. A procedure has been suggested for the design of two-dimensional array excitation coefficients from the linear array coefficients.
Pattern switching concept opens up new possibilities of reducing the effect of sidelobes in weather as well as target detection radars via signal processing technique. More work needs to be done to evaluate the full potential of pattern switching concept vis-a-vis the conventional approach of low sidelobe antenna design.

\section{ACKNOWLEDGMENT}

We appreciate the help of Yosefa Gal-Chen in the initial use of the computer. Michelle Foster and Joy Walton typed the manuscript, and the graphic services were provided by Joan Kimpel and Bob Goldsmith.

\section{REFERENCES}

[1] M. J. Istok, "An analysis of the relation between Doppler spectrum width and thunderstorm turbulence," CIMMS Rep. 23, May 1983.

[2] R. J. Doviak and D. S. Zrnić, Doppler Radar and Weather Observations. New York: Academic, 1984.

[3] P. H. Hildebrand and P. S. Sekhon, "Objective determination of the noise level in Doppler spectra," J. Appl. Meteor., vol. 13, pp. 808$811,1974$.

[4] J. D. Kraus, Antennas. New York: McGraw-Hill, 1950

[5] J. A. Nelder and R. Mead, "Simplex method for function minimization," Comput. J., vol. 7, pp. 308-313, 1965.

M. Sachidananda, for a photograph and biography please see page 293 of the March 1983 issue of this TRANSACTIONS.

Richard J. Doviak (S'50-M'57-SM'72), for a photograph and biography please see page 689 of this issue.

Dusan S. Zrnić (M'73-SM'77), for a photograph and biography please see page 689 of this issue. 